\title{
Electrical and Anatomical Imaging of Arrhythmogenic Substrates for Scar-related Ventricular Tachycardia
}

\author{
Omar A. Gharbia ${ }^{1}$, Susumu Tao ${ }^{2}$, Albert C. Lardo ${ }^{2}$, Henry Halperin ${ }^{2}$, Linwei Wang ${ }^{1}$ \\ ${ }^{1}$ Rochester Institute of Technology, Rochester, NY, USA \\ ${ }^{2}$ Johns Hopkins Hospital, Baltimore, MD, USA
}

\begin{abstract}
Sustained ventricular tachycardia (VT) often involves a reentry circuit formed by narrow channels of surviving tissue within the scar. Catheter ablation is an effective technique to intercept the circuit by targeting these critical channels. The success of VT ablation relies on our ability to assess the mechanism of the VT circuit and identify the location of the ablation targets. This study aims to analyze the VT substrate using high-resolution anatomical and electrical imaging techniques including contact EGM mapping, contrast-enhanced cardiac magnetic resonance (CMR) imaging, and noninvasive electrocardiographic (ECG) imaging. The results indicate that joint electro-anatomical analysis could reveal critical isthmus of the VT circuit. In addition, ECG-imaging was able to identify sites of a scar and signal fractionation visually consistent with the other two modalities, and the reconstructed VT circuit revealed an exit around the critical site identified by combined CMR-EGM data.
\end{abstract}

\section{Introduction}

Scar-related ventricular tachycardia (VT) is a common cause of sudden cardiac death. These life-threatening VT episodes often involve a short circuit that is thought to be formed by narrow strands of surviving tissue inside myocardial scar. Catheter ablation is an effective procedure that treats scar-related VT by intercepting the reentrant pathways within the scar using radiofrequency energy [1].

The success of VT ablation relies on our ability to assess the mechanism of the VT circuit and identify the location of the ablation targets. This requires knowledge about the morphology of the arrhythmia circuit and the heterogeneous structure of the arrhythmogenic substrate. Currently, these data could be obtained by several electrical mapping or structural imaging techniques, each unfortunately providing only a partial view of the complete picture.

The state of the art in VT ablation relies on invasive catheter mapping that sequentially constructs an electrical map of the heart through hundreds of contact sites. To map the VT circuit, therefore, this technique requires the VT to present a distinct and stable morphology for a sufficient amount of time. As a result, although this technique can provide a relatively precise delineation of the critical components of the VT circuit, it is not possible in up to $90 \%$ of the patients due to poorly-tolerated VT or multiple VT morphologies [2]. Alternatively, contact mapping can be done during native rhythm to identify the myocardial scar as low-voltage areas $(\leq 1.5 \mathrm{mV})$ [3]. Recent research also showed that local abnormal electrograms, such as fractionated, late, or double potentials, are related to critical locations of the reentrant circuit [4]. However, data from contact mapping is limited to the sites of catheter contact from one surface at a time (most often endocardium), providing a limited view of the 3D arrhythmogenic substrates.

Cardiac magnetic resonance (CMR) imaging has also been increasingly explored for guiding VT ablation, mainly owing to its ability to image the 3D heterogeneous structure of myocardial scar [5]. However, by the nature of structural imaging, CMR provides little to no data on the morphology of the arrhythmia circuit. Therefore, with CMR alone, it is difficult to extract from the gray zone the sites that are critical to the formation of the VT circuit.

Recently, noninvasive electrocardiographic imaging (ECGi) has been explored for mapping scar-related VT. ECGi is a noninvasive electrical mapping technique that computationally reconstructs cardiac potentials from highdensity body-surface ECG data. With its noninvasive nature, ECGi was shown to be able to map unstable or polymorphic VT within just a few beats; during native rhythm, ECGi was shown to be able to map the scar substrates and potential critical sites with fractionated potential in a completely non-invasive manner [5]. Furthermore, recent studies demonstrated the potential of ECGi to map scar-related VT throughout the epicardial and endocardial layers [6]. However, to date, VT application of ECGi is limited to two case studies of animal models [7] and several smallsized human subjects [8]. One critical obstacle to the ECGi investigation of scar-related VT is the difficulty to obtain quality reference data to validate ECGi results.

The goal of this study is to combine complementary 


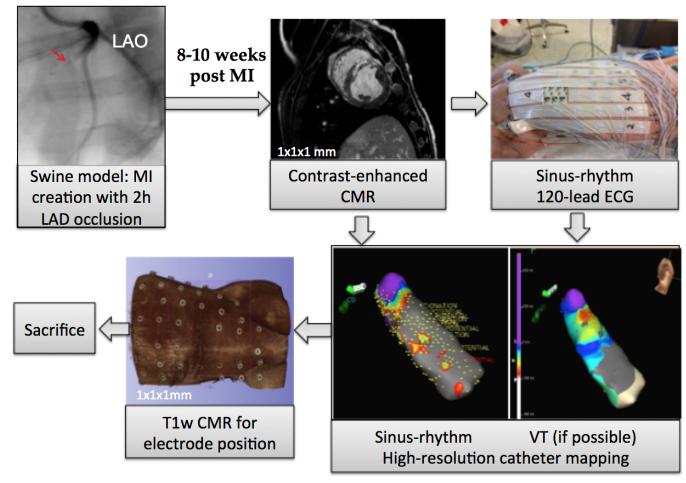

Figure 1. Shows the Workflow of ECGi.

electrical mapping and structural imaging data - in specific, contrast-enhanced CMR imaging, contact mapping, and ECGi - to study the arrhythmia circuit and arrhythmogenic substrates in scar-related VT. The combined CMR and contact mapping data will also provide valuable data for evaluating ECGi solutions. In specific, on post-infarction swine models, we combine high-resolution CMR scar imaging and high-resolution contact mapping to delineate the arrhythmogenic substrates. We then study ECGi-reconstructed arrhythmia circuits with respect to these substrates. This paper reports a case study from our experiments.

\section{Experimental Procedure}

Fig. 1 outlines the experimental procedure. All animal experiments were approved by the Institutional Animal Care and Use Committee.

Animal Model Creation: Swine models of 35-45 kg were used. Prior to any invasive procedure, swine were anesthetized with mechanical ventilation using a combination of tiletamine, zolazepam, ketamine, and xylazine and maintained under sedation using 1-2\% isoflurane. MI was created by inserting a guiding catheter into the left coronary artery and occluded left anterior descending coronary artery just past the first diagonal for two hours using an angioplasty balloon [9].

CMR Imaging: At 8-10 weeks after MI, contrastenhanced CMR imaging was performed on a 3-T scanner (Prisma, Siemens Healthcare). Late gadolinium enhancement CMR was performed 20-30 minutes after injection of Gadopentetate dimeglumine $0.20 \mathrm{mmol} / \mathrm{kg}$. Imaging parameters for free-breathing navigator-gated threedimensional inversion recovery $\mathrm{T} 1 \mathrm{w}$ sequence were: $\mathrm{TI}$ $=400 \mathrm{~ms}$, flip angle $=25, \mathrm{TR} / \mathrm{TE}=5.4 / 2.7 \mathrm{~ms}$, reconstructed pixel size $1.1 \times 1.1 \times 1.1 \mathrm{~mm}$ with interpolation in the slice direction, 12 segments per imaging window, GRAPPA acceleration factor $=2, \mathrm{FOV}=300 \times 220 \mathrm{~mm}$, matrix $272 \times 200$, bandwidth $=200 \mathrm{~Hz} / \mathrm{Pixel}$, scan time $=$ 15-20 min.
Electrophysiology Study: One week after CMR, an electrophysiological study was performed. In heparinized animals, endocardial mapping of the left ventricle was performed during sinus rhythm via a retrograde approach, using a duodecapolar (20-electrode) catheter (AfocusII, 12.5-1mm interelectrode spacing, 1-mm electrode size, St. Jude Medical, Minnetonka, MN) with the NavX mapping system (EnSite Velocity, St. Jude Medical). In addition, a decapolar catheter was advanced from the right jugular vein to the coronary sinus. Field scaling was applied for all mapping. After electroanatomical mapping, to induce VT, programmed ventricular stimulation was performed from two right ventricular sites with up to three extrastimuli decremented to ventricular refractoriness or $250 \mathrm{~ms}$ at two drive trains (600 and $400 \mathrm{~ms}$ ).

ECGi: 120 disposable radiolucent $\mathrm{Ag} / \mathrm{AgCl}$ surface electrodes in 18 strips were placed on the swine torso immediately prior to the electrophysiology study. Bodysurface ECG were recorded during sinus rhythm using the standard Dalhousie mapping protocol [10] on a commercial acquisition system (Active Two, BioSemi, Amsterdam). Afterwards, approximately 10 surface electrodes were removed to accommodate the placement of NavX pads. The rest of the electrodes remained attached throughout the electrophysiology study to record surface ECG during induced VT.

After electrophysiological study and 120-lead ECG recording, the animal was sent to $\mathrm{MR}$ imaging to detect the location of surface electrodes. Imaging parameters for a T1-weighted gradient echo sequence with fat suppression were: reconstructed pixel size $1.1 \times 1.1 \times 1.1 \mathrm{~mm}, \mathrm{FOV}=$ 400x400 mm, matrix 384x307, bandwidth $=620 \mathrm{~Hz} / \mathrm{Pixel}$.

\section{Data Analysis}

CMR scar analysis: 2D stacks of contrast-enhanced CMR images were analyzed using the ADAS-VT software (Galgo Medical, Barcelona, Spain), which delineates the detailed regions of myocardial scar core and gray zone at $10 \%-90 \%$ layer of the ventricular wall at a $10 \%$ interval. Fig. 2 shows examples of the scar map obtained from ADAS-VT at $10 \%, 60 \%$, and $90 \%$ of the pig heart.

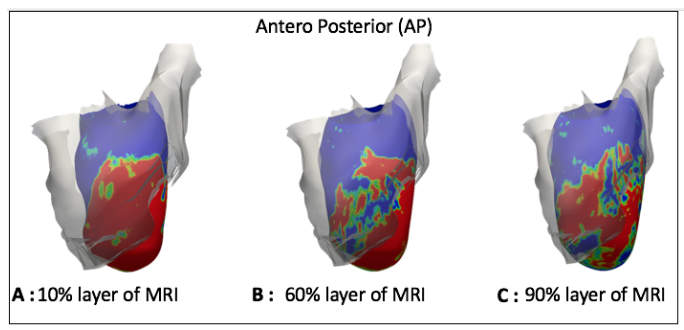

Figure 2. Scar map obtained from ADAS-VT at 10\%, $60 \%$, and $90 \%$ of the ventricular wall. 


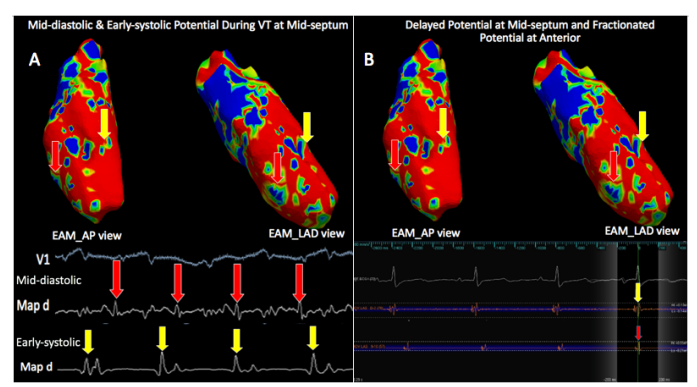

Figure 3. A: Early-systolic and diastolic potential during VT. B: Delayed potential at Mid-septum and fractionated

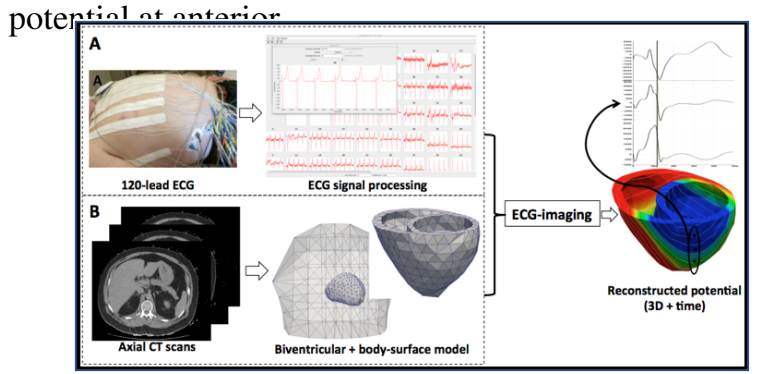

Figure 4. EGI-pipleline A: 120-lead ECG. B: Construction of patient-specific model. From these data, ECGimaging generates eipcardial and endocardial EGMs

Voltage data analysis: On sinus-rhythm voltage data, regions of scar and border zone were first determined using bipolar voltage data with standard clinical thresholds, where normal myocardium voltage $>1.5 \mathrm{mV}$, scar $<0.5$ $\mathrm{mV}$, and border zone (BZ) $0.5-1.5 \mathrm{mV}$. A tiered decreasing of this voltage threshold as described in [11] was then used to reveal potential higher-voltage channels inside the lowvoltage region. In addition, local abnormal electrograms were sought from both sinus rhythm and induced VT data to identify potential critical sites inside the scar. In specific, as the example shown in Fig.3A, sites of fractionated and late potentials during sinus rhythm were identified as potential critical sits. Sites of early-systolic or diastolic potential during VT were also identified (Fig.3B).

ECGi analysis: Fig. 4 outlines the ECGi pipeline used in this study. 120-lead ECG was processed using custom research software (ECGViewer, Rochester Institute of Technology, Rochester, NY). Triangulated mesh of the heart and torso were derived from the CMR images. Given the geometrical models, a forward operator that relates ventricular epicardial and endocardial electrograms to body-surface ECG was obtained using the open-source SCIRun toolkit [12]. The inverse solution was calculated at each time instant using a simple second-order Tikhonov regularization to obtain electrograms on the both epicardium and endocardium:

$$
\hat{s}=\operatorname{argmax}\left\{\|\phi-H s\|_{2}^{2}+\lambda\|\phi-L s\|_{2}^{2}\right\}
$$

where $s$ represents unknown potential on the heart, $\phi$ represents the body surface potential, and $\mathrm{H}$ is the transfer matrix constructed from the heart and torso geometry. $\mathrm{L}$ is a Laplacian matrix on the ventricular surface.

Electrograms for scar substrate were reconstructed from ECG data recorded during sinus rhythm. Scar regions were defined based on EGMs amplitude: Q-wave and R-wave. Critical sites within the scar were defined based on fractionation of EGMs, where the QRS of each signal has lowamplitude and multiple components.

Electrograms for VT circuits were reconstructed during induced VT. Phase mapping based on Hilbert transform was applied to ECG-reconstructed electrograms to track the spatiotemporal evolution of the VT circuit. Activation time was extracted from the phase signals as the time the phase jumps from $\pi$ to $-\pi$, and the site of earliest activation was determined as the VT exit.

\section{4. $\quad$ Results}

Substrate Mapping: As shown in Fig.F5, on this swine, CMR data revealed a septal scar that extends towards the anterior and inferior side of the LV. Voltage data from NavX were qualitatively similar to CMR data, although the extent of the scar was larger compared to that of CMR, especially in the lateral region of the LV. ECGi scar maps were consistent with these data, where the scar size was less extensive than that of $\mathrm{NavX}$ and was more consistent with CMR data.

Within the myocardial scar, as shown in Fig.6A, a channel of surviving tissue was identified by the CMR data on the antero-septal region of the LV. At the same time, middiastolic potential during VT was found at mid-septum of the LV and early-systolic potential during VT was found at antero-septum of the LV, as shown in Fig.6B. It suggested the existence of a critical isthmus at a location consistent to the channel seen in CMR. Fig.6C shows the results form ECGi, where sites of potential fractionation were found at antero-septum and septum of the LV, visually consistent with the critical sites suggested by CMR and voltage data.

VT Mapping: Fig.7 lists the sequence of phase maps

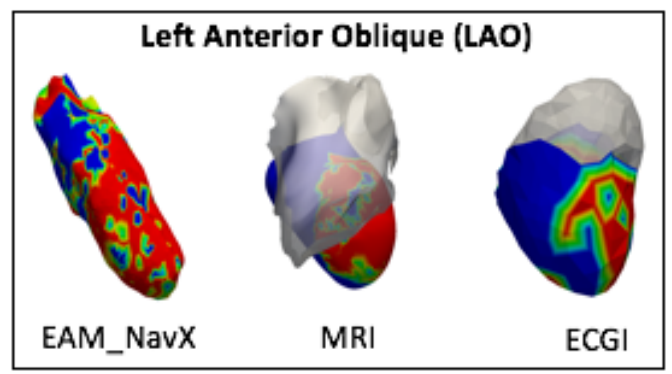

Figure 5. Comparison between CMR, NavX voltage, and ECGI-mapping data. 


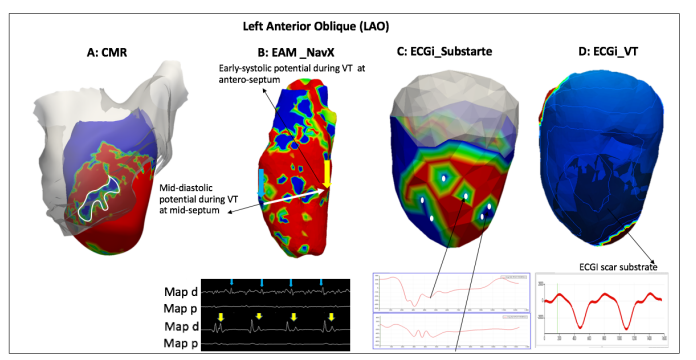

Figure 6. Shows the exist site using activation map derived from the phase map sequence.

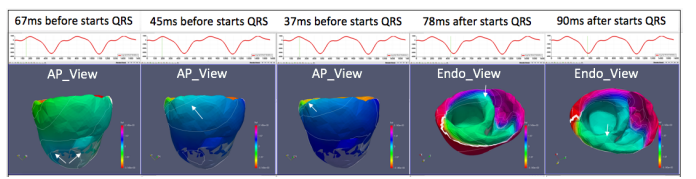

Figure 7. Phase mapping shows the activation of the VT circuits and how it propagates over the heart throughout the epicardum and endocardium.

derived from ECGi-reconstructed VT circuits. As shown, the activation starts at the antero-septum of the LV at 67 ms prior to the onset of the QRS, at a location that is near to the ECGi-imaged scar region. This activation spreads towards the anterior and inferior base of the LV, while a major conduction block was seen along the anterior base of the heart. At the same time, the activation continues through the inferior base of the heart and then the endocardium of the heart, until it meets the conduction block at the anterior base.

Fig.6D summarizes the activation map derived from the phase map sequence, where an exit is seen at the anteroseptum of the LV. This exit site was consistent with the site of fractionation at the margin of the myocardial scar as identified by ECGi during sinus-rhythm substrate mapping, as well as the critical sites as suggested by combined CMR and voltage data.

\section{Conclusions}

Our results showed that combined anatomical and electrical mapping may help assess the arrhythmogenic substrate in relation to the arrhythmia circuit, which could be helpful in assessing the mechanism of VT and identifying the ablation target. Our results also showed that the ECGi technique was able to identify sites of the scar and surviving tissue inside the scar during the sinus rhythm and locate early depolarization sites during the VT. These findings will be further verified in more animal models.

\section{Acknowledgements}

This work was supported by the National Institute of Heart, Lung, and Blood of the National Institutes of Health under Award R21H1125998.

\section{References}

[1] Janse MJ, Wit AL. Electrophysiological mechanisms of ventricular arrhythmias resulting from myocardial ischemia and infarction. Physiol Rev 1989; 69:10491169.

[2] E. Wissner et al., Catheter ablation of ventricular tachycardia in ischaemic and non-ischaemic cardiomyopathy: where are we today? A clinical review, Eur. Heart J., vol. 33, no. 12, pp. 14401450, Jun. 2012.

[3] Ajijola et al., Ventricular Tachycardia in Ischemic Heart Disease Substrates. Indian Heart Journal 66.Suppl 1 (2014): S24S34. PMC. Web. 15 Sept. 2017.

[4] A. Arenal et al. Tachycardia-related channel in the scar tissue in patients with sustained monomorphic ventricular tachycardias: influence of the voltage scar definition Circulation, 110 (2004), pp. 2568-2574.

[5] E Perez-David et al., Noninvasive identification of ventricular tachycardia-related conducting channels using contrastenhanced magnetic resonance imaging in patients with chronic myocardial infarction: comparison of signal intensity scar mapping and endocardial voltage mapping. J Am Coll Cardiol 2011;57:184194.

[6] L. Wang et al., Noninvasive Epicardial and Endocardial Electrocardiographic Imaging of Scar-related Ventricular Tachycardia, Journal of Electrocardiology, vol. 49, no. 6, pp. 887893, 2016.

[7] J. E. Burnes et al., A noninvasive imaging modality for cardiac arrhythmias, Circulation, vol. 102, no. 21, pp. 21522158, 2000.

[8] Y. Wang et al., Noninvasive Electroanatomic Mapping of Human Ventricular Arrhythmias with Electrocardiographic Imaging, Science Translation Medicine, vol. 3, no. 98, p. 98ra84, 2011

[9] E Sosa et al., Epicardial mapping and ablation techniques to control ventricular tachycardia. J Cardiovasc Electrophysiol 2005; 16:449452.

[10] C. L. Hubley-Kozey et al., Spatial features in body-surface potential maps can identify patients with a history of sustained ventricular tachycardia, Circulation, vol. 92 , no. 7 , pp. 18251838, Oct. 1995.

[11] S. Jamil-Copley et al., Application of Ripple Mapping to Visualise Slow Conduction Channels Within the InfarctRelated Left Ventricular Scar, Circulation Arrhythmia and Electrophysiology, p. Epub ahead of print, 2014.

[12] Burton, B.M., Tate, J.D., Erem, B., Swenson, D.J.,Wang, D.F., Steffen, M., Brooks, D.H., Van Dam, P.M., and Macleod, R.S., 2011. A toolkit for forward/inverse problems in electrocardiography within the SCIRun problem solving environment, Proc. of Annual International Conference of the IEEE Engineering in Medicine and Biology Society, pp. 267270.

Address for correspondence:

Name: Omar Gharbia

Full postal address: 147 West Squire Dr., Rochester, NY, 14623

E-mail address: oag1929@rit.edu 